\title{
Shear-driven size segregation of granular materials: Modeling and experiment
}

\author{
Lindsay B. H. May, ${ }^{1}$ Laura A. Golick, ${ }^{2}$ Katherine C. Phillips, ${ }^{2}$ Michael Shearer, ${ }^{1}$ and Karen E. Daniels ${ }^{2}$ \\ ${ }^{1}$ Department of Mathematics, North Carolina State University, Raleigh, North Carolina 27695, USA \\ ${ }^{2}$ Department of Physics, North Carolina State University, Raleigh, North Carolina 27695, USA
}

(Received 15 November 2009; published 5 May 2010)

\begin{abstract}
Granular materials segregate by size under shear, and the ability to quantitatively predict the time required to achieve complete segregation is a key test of our understanding of the segregation process. In this paper, we apply the Gray-Thornton model of segregation (developed for linear shear profiles) to a granular flow with an exponential shear profile, and evaluate its ability to describe the observed segregation dynamics. Our experiment is conducted in an annular Couette cell with a moving lower boundary. The granular material is initially prepared in an unstable configuration with a layer of small particles above a layer of large particles. Under shear, the sample mixes and then resegregates so that the large particles are located in the top half of the system in the final state. During this segregation process, we measure the velocity profile and use the resulting exponential fit as input parameters to the model. To make a direct comparison between the continuum model and the observed segregation dynamics, we map the local concentration (from the model) to changes in packing fraction; this provides a way to make a semiquantitative comparison with the measured global dilation. We observe that the resulting model successfully captures the presence of a fast mixing process and relatively slower resegregation process, but the model predicts a finite resegregation time, while in the experiment resegregation occurs only exponentially in time.
\end{abstract}

DOI: 10.1103/PhysRevE.81.051301

PACS number(s): 45.70.Mg, 47.57.Gc, 81.05.Rm, 64.60.ah

\section{INTRODUCTION}

Granular materials have long been known to segregate by size, shape, density, and other material properties, whether driven by shear or vibration [1-3]. The case of size segregation in shear flow is particularly important, as it arises in such diverse situations as industrial chute flows, rock avalanches, and rotating tumblers [4-8]. Under such shear, large particles typically rise and small particles descend. The dominant mechanism in such cases is thought to be percolation based, where the granular flow acts as a sieve through which the small particles preferentially fall, but arguments based on kinetic theory have been proposed as well $[9,10]$. A number of groups [11-13] have developed continuum models to describe vertical size segregation within the constant shear-rate flows typical of free-surface avalanches. The aim of this paper is to apply a recent modification [14] of the Gray-Thornton (GT) model [13] to a boundary-driven granular system where the shear rate is a nonlinear function of depth. We quantitatively investigate this generalized GrayThornton (GGT) model's ability to capture experimentally observed segregation dynamics and the associated changes in overall packing density.

We perform experiments on binary granular mixtures of spherical particles confined in an annular Couette geometry (see Fig. 1). The system starts in an unstably stratified state with small particles over large particles and progresses to the reverse, stable, configuration as the lower boundary is rotated. Key advantages of this geometry are the ability to run continuously without the need to feed material, as would be the case for chute flows, and the ability to start and end the experiment in well-defined states. As is commonly the case for sheared granular materials [15], we observe that the horizontal velocity profile is not a linear function of depth as assumed in $[12,13,16]$, but instead decays exponentially away from the shearing surface. Correspondingly, the segregation rate is not uniform, but is higher near the bottom rotating plate. We use high-speed digital imaging and particle tracking to measure the velocity profile $u(z)$ in the experiment and use the resulting fit to an exponential form as input to the GGT model.

Kinetic sieving models of segregation [11-13] are based on the notion that vertical size-segregation is principally caused by small particles preferentially falling into gaps created by the relative motion (shear) of the particles beneath them. Secondarily, the large particles are forced upward in a process sometimes called squeeze expulsion. In particular, Bridgwater et al. [11] argue that the vertical velocity of small particles due to segregation (the segregation velocity) should be proportional to the shear rate $\dot{\gamma} \equiv|\partial u / \partial z|$ as well as some function of the concentration $\phi$ of small particles. In the context of chute flow and avalanches, both Lun [12] and Gray and Thornton [13] assume a segregation velocity which is additionally proportional to the concentration of large particles, $(1-\phi)$, since large particles provide the gaps into which the small particles fall. The choice of a constant of proportionality (known as the segregation rate) comes from an assumption of spatially constant shear rate, whereas for many important granular systems it instead falls off exponentially [15].

Since the local shear rate is set by the local horizontal velocity, we measure $u(z)$ directly from the experiment. In our measurements of the horizontal velocity profile, described in Sec. II, we observe that it falls off exponentially away from the shearing surface, is the same for both particle sizes, and is approximately time-independent except for an initial transient. Using the steady-state velocity profile $u(z)$ to determine the shear rate, we calculate an exact solution to the GGT model, described in May et al. [14] and summarized in Sec. III. This solution specifies the concentration of small 


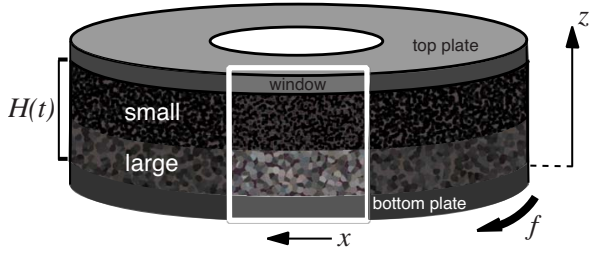

FIG. 1. (Color online) Schematic of experimental apparatus (not to scale) showing initial configuration and coordinate system.

particles as a function of depth and time. Starting from initially segregated conditions, the particles mix via a wave that spreads both downward, as small particles fall, and upward, as large particles rise. After the small particles first reach the bottom plate, they form a growing layer bounded by a shock wave (a discontinuity in concentration) propagating upward. Correspondingly, large particles eventually reach the top of the annulus, and a second shock propagates downward. The two shocks meet in finite time, after which the material is completely resegregated.

While it is not possible to monitor the local concentration field $\phi(z, t)$ within the experiment due to the high packing density, we measure the progression of the mixing and segregation processes via the compaction and subsequent reexpansion of the aggregate. To relate the dynamics of the exact solution to the experiment, we postulate that the local concentration $\phi$ of small particles determines the local packing density $\rho$, as described for static packings in Kristiansen et al. [17]. This concentration map $\rho(\phi)$ allows us to model the change in the measured height $H(t)$ of the aggregate as a function of time. We compare this proxy height $\widetilde{H}(t)$ to the time evolution of the experimentally measured height in Sec. IV.

In Sec. V, we evaluate the successes and failures of the GGT continuum model combined with the dilation model described above. To make a quantitative comparison, we set two of the three free parameters from the observed system height and the magnitude of the transient compaction. The third parameter is the constant of proportionality between shear rate and segregation rate, which we set so that the model and experiment have the same overall duration. This segregation rate has previously been observed to vary with such parameters as particle size ratio and confining pressure [18]. In spite of its simplicity, we find that the GGT model is able to capture the existence of a fast mixing process followed by a slower resegregation process. However, the model resegregates in finite time and does not replicate the observed behavior from the experiment, where a completely resegregated state is approached only exponentially in time.

\section{EXPERIMENT}

The experimental apparatus is an annular Couette cell filled with a bidisperse mixture of spherical glass particles confined by cylindrical walls at inner radius $(25.5 \pm 0.1) \mathrm{cm}$ and outer radius $(29.3 \pm 0.1) \mathrm{cm}$. We apply shear via a circular bottom plate rotating at a constant frequency $f$ $=(49 \pm 0.5) \mathrm{mHz}$, approximately $3 \mathrm{rpm}$. A heavy top plate

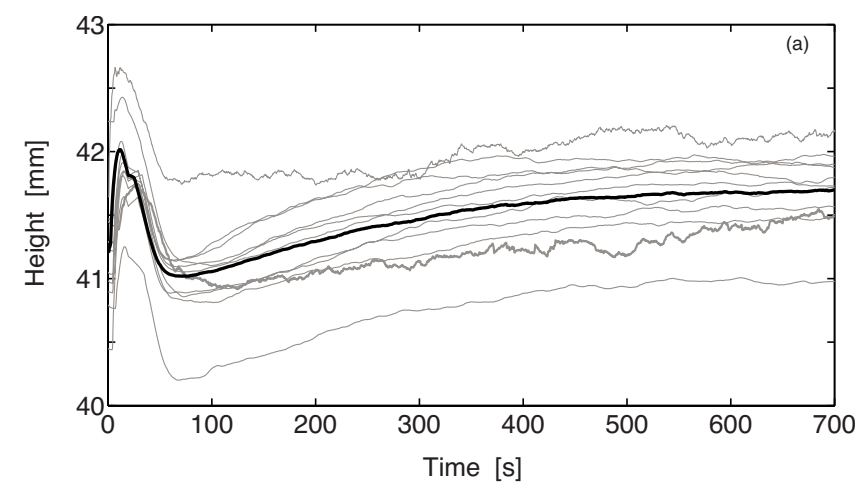

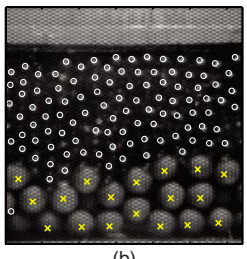

(b)

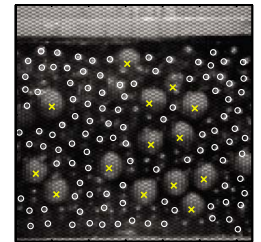

(c)

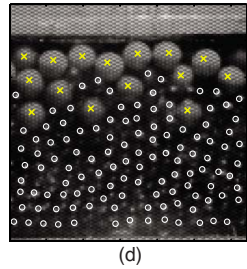

FIG. 2. (Color online) (a) $H(t$ ) for 11 experimental runs (gray lines), with thicker line representing the run for which we took movies and measured the velocity profile. The thick black line is the average of the 11 runs, used for comparison with the GGT model in Fig. 7. Positions of particles $(\bigcirc$ indicates small, $\times$ indicates large $)$ overlaid on images taken through the window in the outer wall (b) initially, $t=0 \mathrm{~s}$, (c) in a mixed state, $t=127 \mathrm{~s}$, and (d) in the resegregated state, $t=1820 \mathrm{~s}$.

sits within the annular gap and is free to move vertically, but is partially suspended by springs to reduce the pressure it applies. The compressive force $P$ applied to the particles is $(0.36 \pm 0.008) \mathrm{mg}$, where $\mathrm{mg}$ is the total weight of the particles and the variation in force is due to the stretching of the springs. The top and bottom plates have a rubberized surface to increase friction with the particles, while the stationary cylindrical side walls are constructed of aluminum.

At the start of each experiment, we prepare a flat layer of $2.0 \mathrm{~kg}$ of large particles (diameter $d_{L}=6 \mathrm{~mm}$ ) at the bottom of the annulus, followed by a flat layer of $2.0 \mathrm{~kg}$ of small particles $\left(d_{S}=3 \mathrm{~mm}\right)$. This configuration is shown schematically in Fig. 1. We consolidate the layers by compression prior to the beginning of the run, with the average initial height $H(0)=41.2 \mathrm{~mm}$. In our experiments, we measure two aspects of the dynamics: the height $H(t)$, and the particle velocities, which create the shear profile responsible for the segregation. In Sec. IV, we discuss a semiquantitative method for relating $H$ to the degree of segregation.

Figure 2 shows the height $H(t)$ of the top of the cell as a function of time for several different runs under the nominally identical conditions. While the variability from run to run is considerable (and typical of granular materials), the important features are common to all of the runs. From the initial normally graded configuration, the applied shear causes the lowest (large) particles to move horizontally due to friction with the rubberized bottom plate. As shear begins, the material must dilate in order to deform (Reynolds dilatancy). After this initial rapid expansion, $H(t)$ provides information about the progression of mixing/segregation in the aggregate. Because the mixing process causes small particles 
to partially fill the voids between the large particles, the total height of the aggregate decreases. For all runs, the height reaches a minimum $H_{\min }$ at $t \approx 80 \mathrm{~s}$, with $\Delta H \approx 1 \mathrm{~mm}$. Further shearing serves to resegregate the particles, with the large particles ending at the top of the cell and the small particles at the bottom. During this process, $H$ rises back to a height greater than its initial value. This resegregation process would in theory continue until there were no longer a mixture of particles in the central region. In practice, however, the particles do not fully resegregate and a few large particles remain within the lower layer, as can be seen in Fig. 2(d). A key advantage of measuring the segregation progress via changes in the volume of the granular materials is that we effectively average over the behavior of $\sim 6 \times 10^{4}$ particles, rather than just the few particles visible in the window.

To measure the velocity of the particles, we observe the outer layer through a window of approximate width $10 \mathrm{~cm}$, using a digital video camera operating at $450 \mathrm{~Hz}$. We recorded images during three time intervals, each with an approximate duration of $10 \mathrm{~min}$ (a total of around $10^{6}$ images), separated by intervals of similar duration during which images were transferred from the camera to the computer. The system reaches a resegregated state after approximately $t_{f}=700 \mathrm{~s}$.

From each image, we first identify the center of each particle by convolution with a circular kernel chosen to match either $d_{S}$ or $d_{L}$. We perform the convolution twice (once for each particle-diameter) and then screen for misdetections and double detections. Figure 2 shows particle centers for (b) particles in the initial, normally graded configuration, (c) the mixed state, and (d) the resegregated, inversely graded state. Because the particular configuration visualized at the wall only measures the state of a small portion of the system, we use the images only for calculating the velocity profile, and use $H(t)$ to probe the average degree of mixing/segregation.

To characterize the shear, we are primarily interested in the average horizontal velocity $u$ and how it depends on depth, time, and particle size. We assemble particle trajectories from the list of particle positions associated with each image, considering each of the two sizes separately. For each trajectory, we calculate the instantaneous velocity of the particle from the slope of a linear fit over a duration appropriate to the average speed of the layer. This analysis was repeated separately for each of the three $10 \mathrm{~min}$ intervals to check for time independence; we include all images with $t<3100 \mathrm{~s}$ in order to improve our statistics. We observed a steady-state velocity profile for all three intervals (shown in Fig. 3), after an initial $\approx 37 \mathrm{~s}$ transient which is excluded from the analysis. We observed that $u(z)$ was approximately the same for both large and small particles.

We measure the average horizontal velocity as a function of depth by dividing the ensemble of trajectories into a discrete set of bins centered at positions $z_{i}$. Within each bin, we plot a probability distribution of the velocities and fit a parabola to the peak. This peak value is a good estimate for the mean of the (symmetric) velocity distribution, and has the advantage of being insensitive to false detections at small values of $u$ which would otherwise skew the average. This maximally likely velocity, $u\left(z_{i}\right)$, is plotted in Fig. 3, with the width of the distribution at half the height of the peak represented by the horizontal bars for each $z_{i}$.
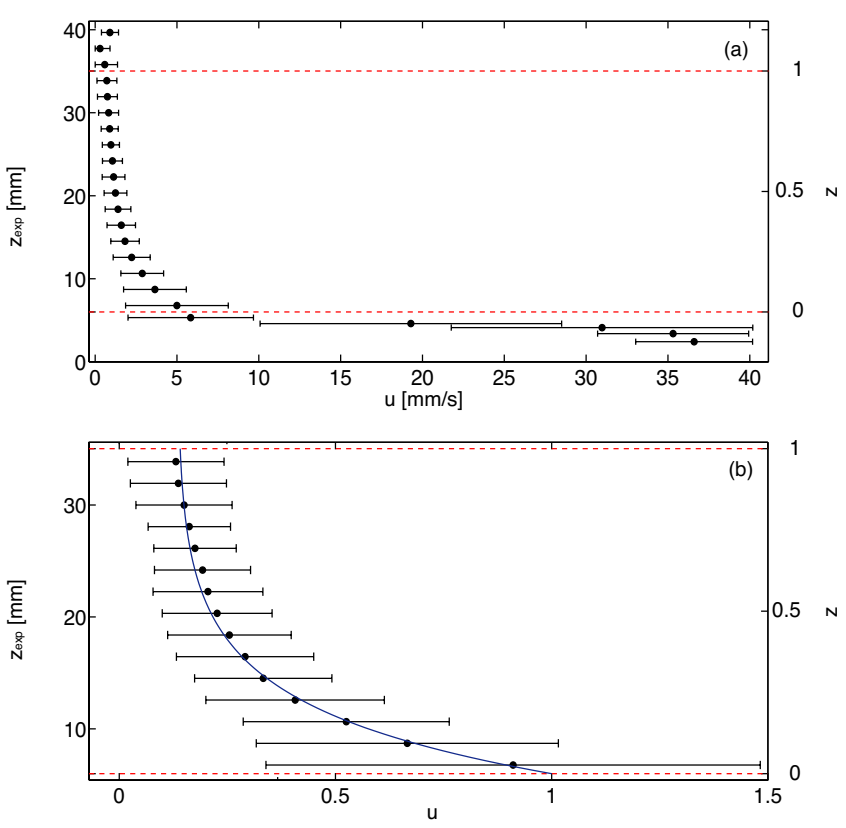

FIG. 3. (Color online) (a) Measured velocity profile $u\left(z_{i}\right)(\mathbf{O})$ for full cell height, with boundary layers of thickness $d_{L}$ above and below the dashed horizontal lines which bound the modeled region. Nondimensional height variable $z$ is scaled so that $z=0$ at the bottom dashed line and $z=1$ at the top line. (b) Dimensionless $u\left(z_{i}\right)$ with velocities scaled by $u=5.5 \mathrm{~mm} / \mathrm{s}$ so that $u(0)=1$. The dashed lines correspond to those in (a). The solid curve is the fit to Eq. (1). Bars represent width of the velocity distribution at half the height of the peak.

Since the segregation is driven by shear in the GGT model, we calculate the experimental shear rate $\dot{\gamma}(z)$ $\equiv|\partial u / \partial z|$ from velocity data by finite differences, shown in Fig. 4. Note that the bottom and top layers, of depth approximately $d_{L}$, show dramatically higher shear rates than the bulk. As a result, these regions quickly accumulate small and large particles, respectively. In the figures, we mark the boundaries between the bulk and the two layers by horizontal dashed lines. In order to model the long-term mixing and resegregation, we focus on the central region of the flow where the segregation process is slower. Since the top and bottom layers form quickly, there is little flux into or out of this region. For comparison with the model, we scale the $z$ axis so that $z=0$ at the bottom of this central region and $z=1$ at the top. Within this region, we observe that the velocity profile is well described by an exponential of the form

$$
u(z)=u_{0} e^{-z / \lambda}+c,
$$

where $\lambda$ is related to the width of the shear band, $u_{0}+c$ is the velocity at $z=0$, and $c$ is a constant representing solidbody rotation. The fit to this form is plotted as a solid line in Fig. 3(b), scaled so that $u(0)=1$. The fit parameters are $\lambda=(0.205 \pm 0.01), u_{0}=(0.87 \pm 0.04)$, and $c=(0.13 \pm 0.07)$.

To obtain $\dot{\gamma}(z)$ for use in the model, we differentiate $u(z)$ in Eq. (1) and find 

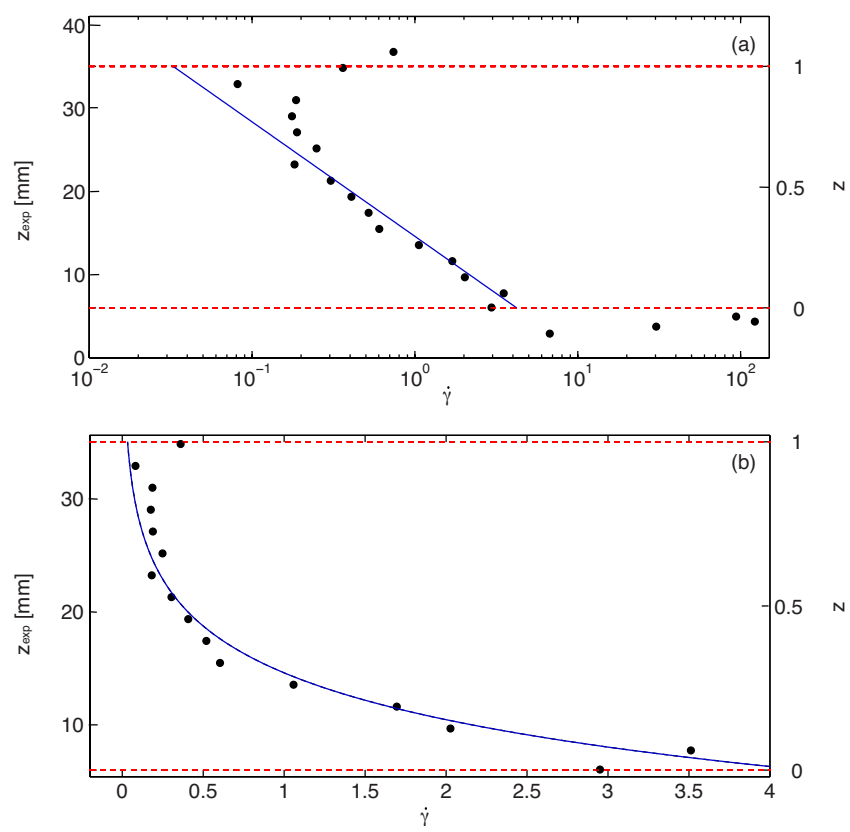

FIG. 4. (Color online) (a) Dimensionless shear rate $\dot{\gamma}=|d u / d z|$ O) on logarithmic axis over full cell height, with dashed lines showing same boundary layers as in Fig. 3. (b) $\dot{\gamma}=|d u / d z|$ within the region $z \in[0,1]$, on linear axes. Solid line is the fit from Fig. 3, plotted as Eq. (2).

$$
\dot{\gamma}(z)=\frac{u_{0}}{\lambda} e^{-z / \lambda} .
$$

This function is plotted in Fig. 4(b) for comparison with the experimentally determined profile obtained by finite differencing. In Sec. III, we will relate the local segregation rate to the parameters $u_{0}$ and $\lambda$.

\section{SEGREGATION MODEL}

We aim to evaluate the ability of the GGT model [14] to describe the segregation dynamics in these experiments. This continuum model consists of an equation for the conservation of mass of each particle size, but no packing density $(\rho)$ or particle length scale. Instead, each point in space is simultaneously occupied by both a concentration $\phi$ of small particles and a concentration $1-\phi$ of large particles, as in mixture theory. A key length scale comes from the width of the shear band (a few particle diameters); the time scale is set by the segregation rate and the vertical size of the system. However, real granular systems compact and dilate during the segregation [as seen in Fig. 2(a)]. Additionally, granular shear bands are known to exhibit a lower packing density than the bulk. Due to a lack of a physical packing density $\rho$, the GGT model cannot capture such dynamics. Thus, in Sec. IV, we propose and evaluate a method for modeling the physical packing density $\rho$ via its relationship to $\phi$. This allows us to relate spatial variations in $\phi$ to changes in the volume of the sample as a function of time. Since the horizontal cross section of the sample is constant, the volume predicted by the model can be compared directly to the experimentally measured height $H(t)$ of the top plate.
The GT conservation of mass equation is [13]

$$
\frac{\partial \phi}{\partial t}+\frac{\partial}{\partial x}(\phi u)+\frac{\partial}{\partial y}(\phi v)+\frac{\partial}{\partial z}(\phi w)+\frac{\partial}{\partial z}[q(z) \phi(\phi-1)]=0,
$$

where $\phi=\phi(x, y, z, t)$ is the concentration of small particles at position $(x, y, z)$ and time $t$, the velocity $(u, v, w)$ is the bulk velocity of small particles, and all variables are dimensionless. Kinetic sieving is modeled by a modification $w_{s}$ $=q(\phi-1)$ to the vertical component $w$ of bulk velocity, in which $q=q(z)$ is the local (dimensionless) segregation rate. The equation incorporates the mechanical transport known as kinetic sieving, but does not include diffusion due to random particle motion. Including diffusion would have the effect of smoothing the solution without affecting its overall structure. Since the Cole-Hopf transform used in Gray and Chugunov [19] does not apply to the GGT model, we instead consider the diffusionless case in order to make use of an analytical result [14].

We assume that the components $v, w$ of the bulk velocity (in the $y, z$ directions, respectively) are negligible. That is, we assume there is essentially no motion across the flow, and that the vertical component of velocity of small particles is dominated by the effect of segregation. In the annular Couette geometry of the experiment, the flow is uniform in the $x$ direction (the angular direction); we assume $u=u(z)$ is independent of horizontal position $(x, y)$ and time $t$. Finally, we assume that the segregation rate $q(z)$ depends only on the vertical variable $z$ and is proportional to the shear rate $\dot{\gamma}(z)$,

$$
q(z)=s \dot{\gamma}(z)=q_{0} e^{-z / \lambda}, \quad q_{0} \equiv s \frac{u_{0}}{\lambda} .
$$

We refer to the dimensionless parameter $s$ as the segregation parameter. In practice, it sets a time scale for the segregation, as we discuss below when comparing the model to the experiment. Note that both $q$ and $s$ are proportional to the gravitational acceleration $g$, as described in Gray and Thornton [13]. In previous experiments, we observed that the time scale of both the mixing and segregation processes is a function of particle size ratio and confining pressure [18].

For small $(v, w)$, the model (3) reduces to the scalar conservation law

$$
\frac{\partial \phi}{\partial t}+\frac{\partial}{\partial z}[q(z) \phi(\phi-1)]=0 .
$$

We set an initial condition corresponding to the beginning experimental configuration of a layer of large particles above a layer of small particles,

$$
\phi(z, 0)= \begin{cases}0, & 0<z<z_{0} \\ 1, & z_{0}<z<1\end{cases}
$$

with $z_{0}=\frac{1}{2}$. Boundary conditions

$$
\phi(0, t)=1, \quad \phi(1, t)=0
$$

ensure that there is no flux of the particles through the upper and lower boundaries. 
In May et al. [14], we constructed the solution of Eq. (5)-(7) using the method of characteristics and shock waves. For early times, $\phi$ varies continuously from $\phi=0$ to $\phi=1$ in an expanding region, as the large and small particles mix together. Mathematically, this is a rarefaction wave solution of the PDE, the terminology deriving from rarefaction or expansion waves in gas dynamics [20]. The solution in the rarefaction wave is characterized by a pair of equations

$$
\begin{gathered}
\phi(z, t)=-\sigma_{0} t \phi_{o}\left(1-\phi_{o}\right) e^{-z_{0} / \lambda}+\phi_{o}, \\
e^{z / \lambda}-e^{z_{0} / \lambda}=-\phi_{o}\left(1-\phi_{o}\right) e^{-z_{0} / \lambda}\left(\sigma_{0} t\right)^{2}+\left(2 \phi_{o}-1\right) \sigma_{0} t,
\end{gathered}
$$

where $\sigma_{0}=q_{0} / \lambda$. In these equations, $0 \leq \phi_{0} \leq 1$ labels a specific characteristic at $t=0, z=z_{0} ; \phi_{0}(z, t)$ can be found by solving the quadratic Eq. (8b), and choosing the relevant solution. Then Eq. (8a) is an explicit formula for $\phi(z, t)$.

The rarefaction reaches the bottom and top boundaries when the characteristics $\phi=1, \phi=0$ reach $z=0, z=1$, respectively. This corresponds to the first small particle reaching the bottom plate and the first large particle reaching the top plate. These events occur at times

$$
t_{0}=\frac{\lambda}{q_{0}}\left(e^{z_{0} / \lambda}-1\right), \quad t_{1}=\frac{\lambda}{q_{0}}\left(e^{1 / \lambda}-e^{z_{0} / \lambda}\right),
$$

respectively. Subsequently a layer of small particles grows from $z=0$, and a layer of large particles grows from $z=1$. The interfaces $z=\Gamma_{0}(t), z=\Gamma_{1}(t)$ between the rarefaction and these layers are shock wave solutions of the conservation law (5), and consequently evolve according to the RankineHugoniot condition, which for Eq. (5) is a differential equation for each shock,

$$
\begin{gathered}
\Gamma_{0}^{\prime}(t)=q\left[\Gamma_{0}(t)\right] \phi\left[\Gamma_{0}(t), t\right], \quad t>t_{0}, \quad \Gamma_{0}\left(t_{0}\right)=0, \\
\Gamma_{1}^{\prime}(t)=q\left[\Gamma_{1}(t)\right]\left\{\phi\left[\Gamma_{1}(t), t\right]-1\right\}, \quad t>t_{1}, \quad \Gamma_{1}\left(t_{1}\right)=1 .
\end{gathered}
$$

When these shocks meet, resegregation is complete, and the solution consists of a stationary shock separating the upper layer of large particles from the lower layer of small particles. By mass conservation, the position of the interface is $z=1-z_{0}$. A contour plot of the solution corresponding to parameter values calculated from the experimental data is shown in Fig. 5.

\section{COMPARISON OF MODEL AND EXPERIMENT}

In the experiment, the height $H(t)$ initially increases due to Reynolds dilatancy, then decreases sharply as the material mixes [see Fig. 2(a)]. Subsequently, $H(t)$ increases at a slower rate as the material resegregates. The GGT model has no inherent way of capturing such dynamics, since the field $\phi$ only measures the local concentration of small particles relative to large, not the packing density $\rho$.

In this section, we relate the mixing and resegregation stages in the height evolution to predictions of the model (5)

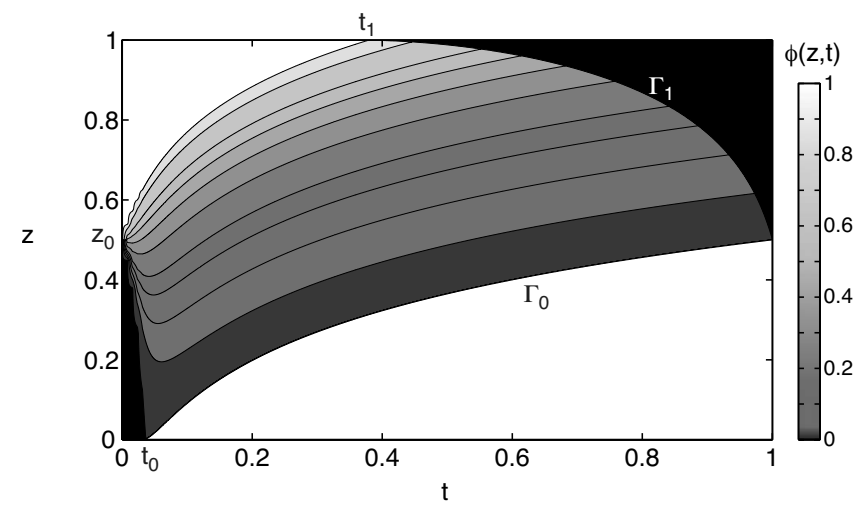

FIG. 5. Numerical solution of the initial value problem Eq. (5)-(7), with parameters $u_{0}=0.866, \lambda=0.205, z_{0}=\frac{1}{2}$. For this plot, we set the segregation parameter $s=16.2$ so that $t=t_{f}=1$ is the (nondimensional) time at the final, fully segregated state.

shown in Fig. 5 by introducing a relationship between $\phi$ and $\rho$. Mathematically, we have a solution $\phi(z, t)$ specified at each point $z$ in the fixed spatial domain $0 \leq z \leq 1$, and at each time $t$. However, the spatial extent of the physical domain varies with time due to the changes in height $H(t)$; the physical vertical coordinate $z H(t)$ must expand and contract to follow these dynamics.

To model changes in $\rho$, we note that a static granular material at different concentrations $\phi$ of small particles will have a different packing density. Monodisperse $(\phi=0$ or $\phi$ $=1$ ) systems pack more loosely than bidisperse mixtures where small particles can partially fill gaps between the large particles. The local packing density $\rho$ is the ratio of the solid local volume occupied by the mixture of particles to the total local volume, i.e., including void space. The total volume of the annular region is the product of the cross sectional area $A$ and the position $H$ of the top plate. For a local solid volume of particles $d V_{p}$ across a cross section of the annulus, in a horizontal layer of small (physical) thickness $d h$, we write

$$
\rho=\frac{d V_{p}}{A d h}, \quad \text { or } \quad d h=\frac{d V_{p}}{A \rho} .
$$

Since the physical domain is expanding and contracting while filled by a fixed volume $V_{p}$ of particles, we can also write $d V_{p}=V_{p} d z$, where $d z$ is the height element in the model domain, $0 \leq z \leq 1$. An alternative reading of this relationship is that for each $d z$ there will be a local contribution to $H(t)$ which depends on the local $\rho$ (which in turn depends on the local $\phi)$.

In order to convert from the model domain $(z)$ to the physical domain (a proxy height $\tilde{H}$ ), we integrate Eq. (11) over the whole system,

$$
\tilde{H}(t)=\int_{0}^{\tilde{H}(t)} d h=\frac{V_{p}}{A} \int_{0}^{1} \frac{d z}{\rho[\phi(z, t)]} .
$$

For simplicity, we have assumed that the local packing density $\rho=\rho(\phi)$ depends only on the local concentration $\phi(z, t)$ and not on other variables. We note that in Eq. (12), $\frac{V_{p}}{A}$ has units of length, and the integral over $d z$ is nondimensional. 

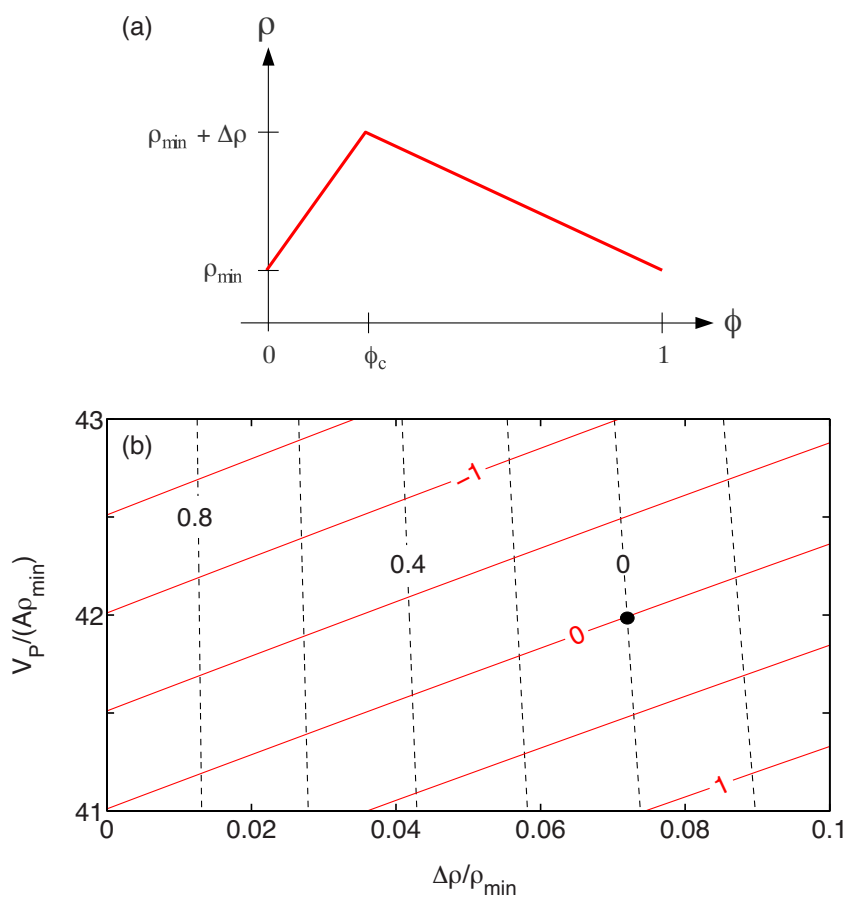

FIG. 6. (Color online) (a) Concentration map specified by Eq. (13). (b) Contour plot of $H_{\min }-\widetilde{H}_{\min }$ (solid lines) and $\Delta H-\Delta \widetilde{H}$ (dashed). Symbol marks the intersection of the two zero contours at $V_{p} /\left(A \rho_{\min }\right)=42.0 \mathrm{~mm}$ and $\Delta \rho / \rho_{\min }=0.072$.

Another way to interpret this equation is that the integral calculates the height of the sample in the mathematical model and the coefficient $\frac{V_{p}}{A}$ relates the model height $\tilde{H}(t)$ to the physical height $H(t)$.

To determine an appropriate function $\rho(\phi)$, we first consider the monodisperse cases in which $\phi=0$ or $\phi=1$. In these cases, the lower limit for $\rho$ is known as random loose packing, $\left(\rho^{R L P} \approx 0.55\right)$ and the upper limit as random close packing $\left(\rho^{R C P} \approx 0.64\right)[21,22]$. However, for bidisperse mixtures, the packing density depends on the relative composition of the mixture. Since small particles can fit within the spaces between large particles, the packing density is larger for a bidisperse mixture than for a monodisperse sample. Indeed, the maximum packing density for a bidisperse mixture with this size ratio has been observed to be around $\rho^{R C P, b i}=0.67$ to 0.69 , depending on the method used $[17,23,24]$.

Data from several numerical and experimental studies $[17,25,26]$ of the random close packing of a static bidisperse mixture of spheres supports an approximately triangular shape [see Fig. 6(a)] for the concentration map $\rho(\phi)$, with a maximum at $\phi_{c}=0.275$. The function

$$
\rho(\phi(z, t))= \begin{cases}\rho_{\min }+\Delta \rho \frac{\phi}{\phi_{c}}, & \phi \leq \phi_{c} \\ \rho_{\min }+\Delta \rho \frac{1-\phi}{1-\phi_{c}}, & \phi>\phi_{c}\end{cases}
$$

falls off, by an amount $\Delta \rho$, to minimum packing density $\rho_{\text {min }}$ at $\phi=0,1$. Kristiansen et al. [17] report a value of $\rho_{\text {min }}$ $=0.628$, which is below both the monodisperse and bidisperse $\rho^{R C P}$.
Note, however, that there is a significant difference between the conditions under which these studies determined the packing density, and the conditions of our experiments: our measurements take place in a sheared system rather than a static one. The packing density for sheared granular materials is typically less than for static packings, due to Reynolds dilatancy. However, we are not aware of any measurements, analogous to those reported in Kristiansen et al. [17], for $\rho(\phi)$ in sheared granular materials. (One can imagine that segregation in fact makes such measurements rather challenging.) Therefore, we must estimate the values of $\rho_{\min }$ and $\Delta \rho$ in the concentration map [Eq. (13)] for our bidisperse mixture of granular materials under shear. We expect that the value of $\rho_{\min }$ may be less than the value found in Kristiansen et al. [17] due to the shear, but is probably not less than the random loose packing limit for monodisperse spheres, $\rho^{R L P}$. Therefore, we consider $0.55<\rho_{\min }<0.64$ as a reasonable range of values. Typical reported values of $\Delta \rho$ range from 0.025 to 0.063 [17]. Since $\rho^{R C P, b i}$ provides a larger upper limit for bidisperse mixtures, we consider a large range of values, $0.02<\Delta \rho<0.14$.

Substituting Eq. (13) into Eq. (12), we observe that there are only two free parameters: $\frac{V_{p}}{A \rho_{\min }}$ sets the overall height of the system and $\frac{\Delta \rho}{\rho_{\min }}$ sets the amount of compaction/expansion. From measurements of apparatus dimensions and particle sizes, we find $\frac{V_{p}}{A}=(24.2 \pm 1.2) \mathrm{mm}$. This range, together with the ranges for $\rho$ and $\Delta \rho$ given above, predict that we should consider parameters $37 \lesssim \frac{V_{p}}{A \rho_{\min }} \lesssim 44$ and $0.07 \lesssim \frac{\Delta \rho}{\rho_{\min }}$ $\lesssim 0.26$. To select the values which best capture the compaction and expansion process, we perform the integral [Eq. (12)] for pairs of $\left(\frac{V_{p}}{A \rho_{\min }}, \frac{\Delta \rho}{\rho_{\min }}\right)$ values, and determine the resulting $\widetilde{H}_{\min }$ and $\Delta \tilde{H}$. In Fig. 6 , we show contour plots of the difference between the experimentally measured values and the proxy-calculated value over this full parameters range. The best parameter choice lies at the intersection of the two zero-contour lines. We find these values to be $\frac{V_{p}}{A \rho_{\min }}=42.0$ and $\frac{\Delta \rho}{\rho_{\min }}=0.072$. The first parameter is to be expected given the height of the system in Fig. 2, and the second is at the lower end of the expected range of values.

A third free parameter is the segregation parameter $s$ in Eq. (5) that effectively sets the time scale for the dynamics. Specifically, we choose $s=0.023$ to scale the ending time for the solution of the initial value problem to agree with the experimentally determined time $t_{f}=700 \mathrm{~s}$. Figure 7 provides a direct comparison of the experiment and the model over this time interval. We observe that the proxy height decreases more rapidly than it climbs, in agreement with the experimentally observed $H(t)$; this feature arises without including separate mixing and segregation rates in the model.

The micromechanical origins of this property remain to be investigated in future work. In addition, the rate at which it decreases is close to the observed rate. At later times, the shapes of $H(t)$ and $\tilde{H}(t)$ no longer agree, with $\widetilde{H}(t)$ exhibiting both a flatter minimum and a faster resegregation rate. This is to be expected from the numerical solution of Fig. 5, since over a substantial portion of the evolution, the width of the rarefaction wave (shaded in the figure), in which the small 


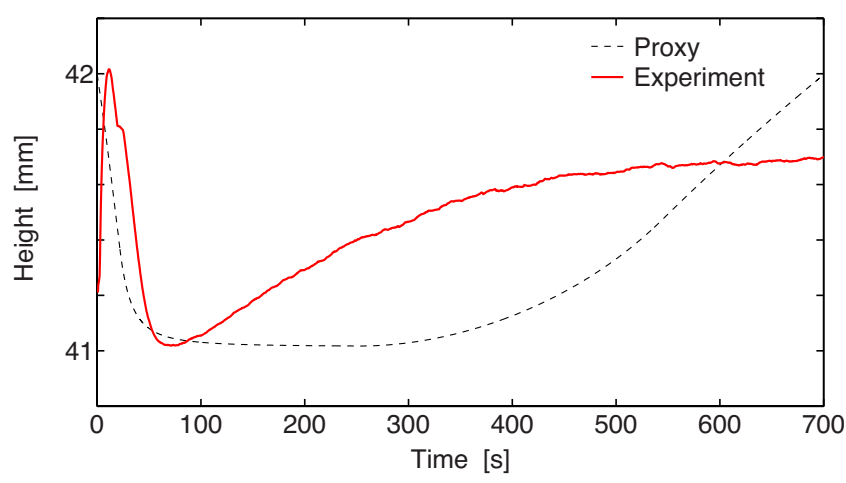

FIG. 7. (Color online) Comparison of the experimentally measured $H(t)$ (solid line, see Fig. 2) and the calculated proxy height $\widetilde{H}$ (dashed line) for the parameters given in Fig. 6.

and large particles are mixed, is not changing significantly. Consequently, the integral (12) giving $\widetilde{H}(t)$ will be nearly constant in time over this part of the evolution, no matter what the choice for the concentration map $\rho(\phi)$. It is particularly notable that the model solution predicts resegregation in finite time, in contrast to the exponential approach to resegregation observed in the experiment [18].

\section{CONCLUSIONS}

We have performed quantitative experiments to compare predictions for a recent extension [14] of the Gray-Thornton model of granular segregation [13] to a flow in which the shear rate is nonconstant. In order to account for the observed dilation, this comparison additionally requires the construction of a model connecting the local concentration of small and large particles to changes in the local packing density (the concentration map). When these two elements are combined, we can compare the temporal evolution of the height of the segregating system to a proxy height calculated from the continuum model. Several features of the experimentally measured height dynamics $H(t)$ are well-captured by the proxy height: (1) a fast mixing time scale is followed by a much slower resegregation time scale, (2) we can model the compaction and re-expansion process using reasonable parameter values in the concentration map, and (3) the slope of the height curve during the mixing phase is in approximate agreement with that of the model.
Some prominent features are missing from the model: there is no means to account for Reynolds dilatancy, and the model segregates in finite time rather than exponentially approaching a final, resegregated state. This latter point of disagreement shows the limitation of using a continuum mixture-theory model for a discrete process, especially where the number of discrete objects (the particles) is comparatively small. In the experiment, it is easy to see how this finite-size effect takes over after the continuum model predicts complete segregation. Once most of the large particles have reached the upper layer, it becomes increasingly difficult for the few large particles remaining in the lower region containing mostly small particles to segregate to the upper layer. It may be that when large particles do not have large particle neighbors, the sieving of small particles is suppressed due to the scarcity of gaps to fall into. In fact, some of the large particles never make it to the upper layer, even after runs of several days duration, instead remaining trapped among the small particles. Even for a larger number of particles, the accuracy of a continuum model might not improve since the width of the shear band scales approximately with the particle size (usually $2-10$ particles). Thus, the fraction of the system contained within the shear band would become negligible in the limit of small particle size. Second, granular materials often fail to self-average: no known length scale exists at which it is accurate to homogenize a dense granular system.

Despite the differences between the dynamics of the experimental and proxy heights, the proxy height calculated from this simple continuum model captures the qualitative features of the experimental height time series. We have extended the Gray-Thornton model, which was developed for the case of an avalanche (uniform shear), to model nonuniform shear, as occurs in a one-dimensional configuration in an annular Couette cell. It is noteworthy that a simple continuum model applied to a small scale granular system successfully captures the main phenomena of mixing and resegregation.

\section{ACKNOWLEDGMENTS}

The authors are grateful to Nico Gray for discussions about the model, and to David Fallest and Dhrumil Patel for initial hardware development and experiments. This research was supported by the National Science Foundation under Grant No. DMS-0604047, and the National Aeronautics and Space Agency under Grant No. NNC04GB086.
[1] J. M. Ottino and D. V. Khakhar, Annu. Rev. Fluid Mech. 32, 55 (2000).

[2] A. Kudrolli, Rep. Prog. Phys. 67, 209 (2004).

[3] M. Schröter, S. Ulrich, J. Kreft, J. B. Swift, and H. L. Swinney, Phys. Rev. E 74, 011307 (2006).

[4] J. A. Drahun and J. Bridgwater, Powder Technol. 36, 39 (1983).

[5] R. M. Iverson and J. W. Vallance, Geology 29, 115 (2001).

[6] N. Taberlet, M. Newey, P. Richard, and W. Losert, J. Stat.
Mech. (2006) P07013.

[7] O. Zik, D. Levine, S. G. Lipson, S. Shtrikman, and J. Stavans, Phys. Rev. Lett. 73, 644 (1994).

[8] D. V. Khakhar, J. J. Mccarthy, and J. M. Ottino, Phys. Fluids 9, 3600 (1997).

[9] S. Hsiau and M. Hunt, Acta Mech. 114, 121 (1996).

[10] D. K. Yoon and J. T. Jenkins, Phys. Fluids 18, 073303 (2006).

[11] J. Bridgwater, W. S. Foo, and D. J. Stephens, Powder Technol. 41, 147 (1985). 
[12] S. B. Savage and C. K. K. Lun, J. Fluid Mech. 189, 311 (1988).

[13] J. M. N. T. Gray and A. R. Thornton, Proc. R. Soc. London, Ser. A 461, 1447 (2005).

[14] L. B. H. May, M. Shearer, and K. E. Daniels, J. Nonlinear Sci. (to be published); e-print arXiv:1002.1979.

[15] GDR MiDi, Eur. Phys. J. E 14, 341 (2004).

[16] J. M. N. T. Gray, M. Shearer, and A. R. Thornton, Proc. R. Soc. London, Ser. A 462, 947 (2006).

[17] K. D. Kristiansen, A. Wouterse, and A. Philipse, Physica A 358, 249 (2005).

[18] L. A. Golick and K. E. Daniels, Phys. Rev. E 80, 042301 (2009).
[19] J. M. N. T. Gray and V. A. Chugunov, J. Fluid Mech. 569, 365 (2006).

[20] R. Courant and K. O. Friedrichs, Supersonic Flow and Shock Waves (Interscience Publishers, New York, 1948).

[21] J. D. Bernal, Proc. R. Soc. London, Ser. A 280, 299 (1964).

[22] G. Y. Onoda and E. G. Liniger, Phys. Rev. Lett. 64, 2727 (1990).

[23] A. S. Clarke and J. D. Wiley, Phys. Rev. B 35, 7350 (1987).

[24] I. Biazzo, F. Caltagirone, G. Parisi, and F. Zamponi, Phys. Rev. Lett. 102, 195701 (2009).

[25] S. M. K. Rassouly, Powder Technol. 103, 145 (1999).

[26] K. Lochmann, L. Oger, and D. Stoyan, Solid State Sci. 8, 1397 (2006). 